\title{
COVID-19: aportes y limitaciones de la enseñanza virtual asíncrona (una experiencia de educación media superior)
}

\section{COVID-19: contributions and limitations of asynchronous virtual teaching (an experience of upper secondary education)}

\author{
SEBASTIÁN SAGARRA ${ }^{1}$ \\ ${ }^{1}$ Centro Regional de Profesores del Sur (Atlántida), Consejo de Formación en Educación, Uruguay. ORCID: 0000-0001-5671-9987 \\ sebasag@montevideo.com.uy \\ Recibido: 15 de setiembre de 2020 - Aceptado: 15 de noviembre de 2021
}

\section{Resumen}

Se indagan aquí los resultados de la transición forzada hacia la enseñanza e-learning, producto de la actual pandemia de COVID-19. Se trata de una investigación contextualizada sobre grupos de enseñanza media superior (EMS) en la asignatura biología. Como base de valoración se consideran indicadores de seguimiento del curso y rendimiento académico del año previo (2019). La comparación interanual muestra que en 2020 un menor número de estudiantes mantuvieron contacto con la asignatura. Entre los que sí participaron, hay evidencias de mayor progreso académico con la puesta en práctica del modelo e-learning asíncrono. En busca de explicaciones para estos incipientes resultados se explora la opinión de los propios estudiantes.

Palabras clave: e-learning, resultados académicos, enseñanza media superior, COVID-19.

\begin{abstract}
The results of the forced transition to e-learning teaching, as a result of the current COVID-19 pandemic, are investigated here. It is a contextualized research on upper secondary education groups (USE) in the biology subject. As a basis for assessment, indicators for monitoring the course and academic performance of the previous year (2019) are considered. The interannual comparison shows that in 2020 a smaller number of students who maintained contact with the subject. Among those, who did participate, there is evidence of greater academic progress with the implementation of the asynchronous e-learning model. In thesearch of explanations for these incipient results, the opinion of the students themselves is explored.
\end{abstract}

Keywords: e-learning, academic results, upper secondary education, COVID-19. 


\section{Introducción}

En respuesta a la pandemia de la enfermedad COVID-19, 107 países han interrumpido la asistencia a las actividades de enseñanza presencial (Viner et al., 2020). Aún sin certeza del efecto de la medida sobre la propagación del SARS-CoV-2, urge evaluar su impacto sobre el desempeño de la educación formal. A nivel de 5to. año de EMS en Montevideo, la presencialidad se suspendió desde el 13 de marzo hasta el 29 de junio de 2020. Este período constituye un "experimento socioeducativo" inédito y no planificado, que permite estudiar la reacción sistémica orientada a dotar de continuidad al proceso de enseñanza.

Ante la emergencia educativa y dada la actual disponibilidad de tecnologías de la información y la comunicación (García, 1999), la alternativa obvia para afrontar la suspensión de clases presenciales fue derivar el quehacer educativo hacia la virtualidad. La transición pudo procesarse en diverso grado y multiplicidad de modalidades, siendo las principales variables a contemplar el carácter síncrono o asíncrono de las actividades, su planificación, y la naturaleza y magnitud del acompañamiento proporcionado por los docentes.

En un trabajo anterior (Sagarra, 2018) se investigó si grupos concretos de segundo año de EMS, se mostraban preparados para afrontar un eventual formato de enseñanza de aula invertida (AI) (Bergmann \& Sams, 2012). Una conclusión provisional indicaba entonces que los estudiantes "evidenciaron aptitudes iniciales necesarias para el trabajo en Al". La actual coyuntura ha permitido explorar el punto con mayor profundidad, analizando el resultado académico de la aplicación durante un semestre de un modelo de enseñanza e-learning asíncrono. Como base de comparación se recurrió a datos del primer semestre de 2019, cuando se empleó una metodología presencial tradicional. A continuación se enuncian los objetivos del trabajo:

- Comparar el seguimiento de los cursos, los resultados académicos y su progresión a lo largo de los primeros semestres 2019 y 2020.

- Relevar las opiniones de los estudiantes de 2020 en relación a la nueva propuesta de trabajo.

- Identificar, a partir de las acciones previas, las fortalezas y debilidades de las dos aproximaciones (presencial y virtual asíncrona) en los grupos de trabajo.

\section{Metodología}

a) Enfoque general

En este trabajo se realizó el estudio de un caso. Se trata de una investigación-acción donde el docente inquiere en el curso de su propia práctica y en la interacción con los estudiantes (Mills, 2003). Exceptuando las opiniones relevadas, se procuró obtener información de carácter cuantitativo, a partir de la cual alcanzar conclusiones sustentadas en evidencias estadísticas. La propia naturaleza de la propuesta inhabilita la selección de los participantes según criterios de muestreo. Por lo tanto, no existe pretensión de extrapolar los resultados a un universo externo de mayores dimensiones. Sí se entiende que las tendencias aquí constatadas pueden resultar de utilidad en la planificación de cursos con formato e-learning, b-learning (Graham, 2006) o Al destinados a grupos similares a los indagados.

b) Grupos de trabajo y diseño de la investigación

La investigación se realizó durante los primeros semestres de 2019 y 2020 en cuatro grupos (dos cada año) de segundo año de EMS diversificación humanística, en la asignatura biología. Ambos años se trabajó en la misma institución localizada en el municipio E de Montevideo ${ }^{1}$. Durante el año 2019 se planificó un curso presencial tradicional, con clases expositivo-dialogadas matizadas por ocasionales presentaciones orales de equipos de estudiantes. Se contó con una página web de referencia con función de repositorio de los materiales del curso (Santos, et al., 2012). Si bien se instó al uso de la página, la enseñanza se centró en el aula y, subsidiariamente, en el trabajo domiciliario por parte de los estudiantes. Las clases de $80^{\prime}$ se dictaron una vez por semana en cada grupo $(N=62)$.

Para 2020 se tenía planificado comenzar el trabajo en formato $\mathrm{Al}$, utilizando la plataforma $\mathrm{CREA}^{2}$ como entorno virtual de aprendizaje -EVA- (Salinas, 2011). Tras dos semanas de iniciados los cursos las circunstancias llevaron a la suspensión de las clases presenciales. Sin dilación se reperfiló la labor hacia un modelo de e-learning asíncrono, capitalizando el hecho de que los estudiantes ya habían comenzado su trabajo en plataforma. En el EVA se puso a disposición de los grupos $(N=67)$, en forma semanal, objetos de aprendizaje cuidadosamente diseñados para

1 Región caracterizada por una tasa de egreso de EMS de $65 \%$ (INEEd, 2017), lo que supera en más de 1,5 veces el promedio nacional.

2 https://ceibal.schoology.com/ 
la preparación de las unidades en forma autónoma. Durante el semestre se utilizaron 13 videos cortos ( $5^{\prime}$ a $20^{\prime}$ de duración), 5 videolecciones de mayor duración ( $20^{\prime}$ a $\left.50^{\prime}\right), 5$ textos de estudio ( 2 a 10 carillas), 5 repartidos de ejercicios y 5 foros de resolución de los mismos, así como 12 cuestionarios de evaluación (además de la prueba diagnóstica y la encuesta de seguimiento del curso virtual). También se suministraron audios y audiovisuales con sugerencias para abordar el estudio de las unidades.

Los objetivos de aprendizaje semestrales fueron idénticos los dos años. Lo que se modificó fue la modalidad de enseñanza. Consecuentemente, se aplicaron pruebas bimensuales similares, una correspondiente a las unidades 1 y 2 (sobre fines de abril) y otra correspondiente a las unidades 3 y 4 (sobre fines de junio). En virtud de su planificación, se asume que todas las pruebas (de los dos años y dentro de cada semestre) tuvieron similar grado de dificultad ${ }^{3}$. Asimismo, se considera que, por efectos estadísticos, los grupos 2019 y 2020 son equivalentes desde el punto de vista de las habilidades y conocimientos previos al inicio del curso. De este modo, las diferencias encontradas en los resultados académicos se interpretan aquí como consecuencia de la metodología empleada en cada caso.

\section{c) Análisis estadístico}

Los datos de las pruebas escritas y los cuestionarios se organizaron y graficaron con Microsoft Excel 2016. Los test estadísticos se realizaron con las aplicaciones online suministradas por el sitio web Social Science Statistics https://www.socscistatistics.com.

\section{Resultados}

De los 67 estudiantes inscriptos en 2020, 61 (91\%) se registró en la plataforma virtual durante el primer semestre. Asimismo, 58 (87 \%) realizó al menos una de las 17 propuestas del período. Más allá de estos datos, el estudio emplea como indicador de seguimiento del curso la realización de las pruebas escritas. En ese sentido, 45 estudiantes (67\%) efectuó al menos una de

3 Además, tanto los escritos presenciales como los virtuales fueron pruebas individuales y se extremaron las medidas disponibles para resguardar la fiabilidad de los resultados, apuntando a evaluar aprendizajes "genuinos". las dos pruebas online ${ }^{4}$. Paralelamente, 53 estudiantes (85\%) ejecutaron al menos uno de los dos escritos presenciales en 2019.

En busca de mayor agudeza estadística, se confrontaron las proporciones de realización de los escritos de 2019 y 2020 (Anexos, figura 1) con pruebas de $\chi^{2}$ (Anexos, tabla 1). Para evaluar el rendimiento estudiantil, se consideraron los resultados de las pruebas escritas (por sobre otras actividades presenciales -2019- o virtuales -2020). Tal simplificación está justificada con el fin de facilitar el manejo y la comparabilidad de los datos. En primer lugar, se presentan los resultados de las pruebas escritas del primer semestre 2019 y 2020 (Anexos, figura 1). Luego, trasladando el procedimiento aplicado en relación al seguimiento del curso, se realizó una prueba de dex ${ }^{2}$ cotejando la proporción de aprobados y no aprobados de ambos años (Anexos, tabla 1).

Completando el análisis previo, se realizó la comparación de medias de las pruebas 2019 y 2020 (Anexos, figura 2). Todas las series de datos cumplieron con el requisito de homocedasticidad evaluado a través de la prueba de Levene. Dos de las series, sin embargo, no superaron el contraste de normalidad de Shapiro-Wilk (datos no incluidos). De todos modos, se procedió a realizar las pruebas $t$ de student (Anexos, tabla 2) por considerar que el apartamiento de la normalidad se debe al tamaño reducido del conjunto de datos. Reafirmando los resultados obtenidos con la prueba $t$, se ensayaron test no paramétricos sobre las mismas series que arrojaron resultados análogos, al nivel de significación indicado $(p<0,05)$-datos no incluidos-

Finalmente, para evaluar la opinión de los estudiantes con respecto al curso virtual, se aplicó un cuestionario semiestructurado (Alcaraz, et al., 2006) de entrega voluntaria y no calificada por la vía del EVA. El mismo fue respondido por 28 estudiantes (42\% del grupo). De ellos, 24 (86 \%) manifestó haberse registrado en plataforma en los meses de marzo (21) y abril (3), los restantes lo hicieron con posterioridad. Frente a la pregunta de si "el curso le ayudó a aprender las unidades temáticas": 4 (14\%) respondieron que los ayudó mucho, 17 (61 \%) que los ayudó en algo, 5 (18\%) que no los ayudó y 2 (7\%) no tienen opinión por haberse unido recientemente a la plataforma. Las respuestas relacionadas con la interacción

4 Desde el retorno a la presencialidad se han planteado actividades de recuperación para los estudiantes que no han seguido en forma adecuada el curso virtual. De todos modos, esa no es la materia del presente ensayo. 
de los estudiantes en el marco del curso virtual se sistematizan en anexos, tabla 3.

\section{Análisis de Resultados}

De la inspección de los datos sobre la realización de las pruebas resulta claro que: a) un mayor número de estudiantes realizó las correspondientes a 2019; b) la proporción entre realizados y no realizados fue significativamente mayor en 2019 para los dos escritos del semestre (Anexos, figura 1 y tabla 1). En síntesis, considerando estos datos como reflejo del seguimiento del curso, resulta innegable que el formato e-learning se mostró menos efectivo a los fines de vincular un mayor número de estudiantes con el curso.

¿Qué causas pueden explicar este comportamiento? Cuando se interroga a los estudiantes sobre los inconvenientes para seguir el curso virtual y los motivos por los cuales no realizaron algunas tareas, las razones evocadas ${ }^{5}$ son de dos tipos: materiales y cognitivo-actitudinales (Anexos, tabla 3). Las primeras relacionadas con inconvenientes de conectividad, informáticos o carencias de hardware. Las segundas refieren a dificultades de comprensión y/o deficiencia de hábitos de trabajo.

Al comparar los rendimientos en las pruebas entre los dos años, no se aprecian diferencias significativas en los promedios del primer escrito de 2019 y 2020. Por contraposición, el segundo escrito 2020 tuvo una media significativamente mayor a la de 2019. Sumado a esto, no hay diferencias significativas entre los resultados del primer y segundo escrito de 2019 y sí se comprueba un rendimiento aumentado en el segundo escrito de 2020 (Anexos, figura 2 y tabla 2). Esto podría estar dando cuenta de un progreso en el desempeño de los grupos de 2020 que en 2019 no se procesó.

Apelando a las opiniones de los estudiantes en busca de posibles causas del avance del grupo en 2020, es posible construir el siguiente relato de conjunto (Anexos, tabla 3): el curso virtual asíncrono permitió realizar consultas, acceder a buenos y abundantes materiales, entre ellos videos donde estudiar y tomar apuntes con mayor tranquilidad que en la clase presencial e involucrando a las familias en el proceso. En oposición

5 Es presumible que los 28 estudiantes que respondieron la encuesta presenten el sesgo pertenecer al grupo de mayor vinculación con el curso. De todos modos, como se aprecia en los datos recogidos, se obtuvo una amplia variedad de opiniones ricas en contenido. coexiste un relato crítico de la virtualidad consignando que:es más difícil entender sin el contacto humano directo con el profesor, sin participar ni poder expresarse y sin recibir la retroalimentación inmediata por parte del docente que contribuya a concentrarse en el estudio.

Es interesante cotejar los resultados académicos del segundo escrito 2020 -35 \% más alto que el de 2019(Anexos, figura 2) con la percepción de si el curso virtual fue peor (63\%), mejor (26\%) o igual (11\%) que uno presencial (Anexos, tabla 3). Más allá de la preferencia mayoritaria por la presencialidad, en su ausencia, el $50 \%$ de quienes respondieron prefirió el trabajo en un EVA frente a un $25 \%$ que se inclinaron por videoconferencias y un $18 \%$ por Whatsapp o e-mail. El restante $7 \%$ sugirió combinaciones de estos medios o de los mismos con la presencialidad. La predilección por el EVA se fundamentó en que: su funcionamiento sencillo, su mejor organización de contenidos y la posibilidad de compartir las participaciones grupales, facilitan el trabajo autónomo y anulan la pérdida de clases. Así se generan hábitos de estudio y se eliminan interferencias con otras actividades personales. Además, se aprecia que la asistencia a las videoconferencias es escasa. Aquellos estudiantes que prefirieron actividades virtuales síncronas, lo hicieron basados en que estas brindan mayor trato con el docente y quienes optaron por Whatsapp o e-mail se fundaron en que la comunicación es más rápida.

\section{Discusión y conclusiones}

La irrupción de la pandemia de COVID-19 puso inesperada tensión al sistema educativo formal. La crisis evidenció debilidades y fortalezas, algunas conocidas o presumibles y otras imprevistas. También extendió la posibilidad de investigar y reflexionar acerca de cómo estudiantes, docentes y centros educativos enfrentaron la contingencia. A partir del conocimiento emergente será posible imaginar escenarios futuros. El presente trabajo constituye un modesto aporte en este sentido. Es el relato documentado de una intervención educativa contextualizada. Como tal, es susceptible de contrastación de sus resultados con los macrodatos derivados de la actual coyuntura u otras investigaciones.

La información aquí recabada muestra que el curso virtual asíncrono tuvo una menor capacidad de retención estudiantil respecto al curso presencial. Está claro que la transición hacia la virtualidad se produjo en forma intempestiva motivada por crisis sanitaria. Quizá un 
e-learning de aplicación más parsimoniosa pueda ser más exitoso en el aspecto retentivo. En contraposición, y a pesar de la percepción estudiantil mayoritaria, los datos indican un progreso académico mayor con la modalidad e-learning. Se podría objetar que el menor número de estudiantes que siguió el curso virtual pudo tener un desempeño mayor al que hubiese logrado la totalidad del grupo (ya sea en la misma u otra modalidad de trabajo). En todo caso, tal efecto no se manifestó en el primer escrito (que tuvo igual resultado que el año previo). Lo que se aprecia con claridad en 2020 es un marcado incremento de la media de la segunda prueba (de allí la apelación al concepto de "progreso"). Este evento no tuvo paralelo en 2019. Parece sensato concluir entonces que, en el aspecto académico, el curso virtual resultó como mínimo igual de válido que el presencial ${ }^{6}$.

Como fue dicho, las dificultades aludidas respecto al seguimiento del curso virtual quedan comprendidas en dos categorías: materiales y cognitivo-actitudinales. Uruguay se caracteriza por una alta conectividad informática (Failache, et al., 2020). Pese a ello, aspectos económicos, técnicos y administrativos son puntos críticos a mejorar para universalizar el acceso a los cursos virtuales. Los motivos cognitivos y actitudinales entrañan una mayor complejidad, fundamentalmente en relación a los hábitos de estudio, perseverancia, motivación, estrategias y competencias previamente adquiridas. Sin duda estos factores operan tanto en la presencialidad como en la virtualidad, pero en esta última resultan más explícitos pues los estudiantes, ante la dificultad o carencia, pueden optar simplemente por "desconectarse".

Es evidente que muchos estudiantes percibieron con claridad las ventajas "de manual" del trabajo virtual asíncrono (Soto, Senra\& Neira, 2009). Incluso una proporción pequeña manifestó preferir esta modalidad. Asimismo, el factor social y el contacto humano directo es echado de menos por la mayoría y parece erigirse en un factor decisivo para la preservación del vínculo de muchos estudiantes con la institución. Esta apreciación tampoco resulta novedosa. Citando a Salomón (2002): "El cultivo de las habilidades, la construcción

6 Esta conclusión se realiza (como todo el trabajo) para el caso particular. Entre otros factores, el curso virtual propuesto contó con una cuidadosa planificación de las unidades, selección de los materiales y calendario de trabajo. No es equiparable a "cualquier" curso virtual. Esta experiencia contó con pautas claras, ritmo de trabajo continuo, presencia permanente del docente y seguimiento meticuloso. del conocimiento significativo y del metaconocimiento requiere un entorno humano que al menos proporcione: a) una activa comunidad de aprendices y profesores y b) seguimiento y tutoría".

Dirigiendo la mirada hacia el futuro, parece adecuado incorporar a las prácticas de rutina los atributos en los cuales cada modalidad exhibe mayor potencia: la presencialidad como dinamizador del relacionamiento, apoyo, consultoría, orientación, tutoría, interacción colectiva, etc. La virtualidad como organizador, repositorio, generador de hábitos, vía de comunicación y facilitador del desarrollo académico. No puede descartarse la posibilidad de generar ofertas de educación virtual, mayoritariamente asíncrona, para aquellos estudiantes que puedan afrontarla, sean más autónomos o se vean favorecidos por las características que este tipo de cursos provee.

El desarrollo científico-tecnológico nos ofrece una multiplicidad de alternativas, presenciales y virtuales, para la configuración de nuevas prácticas educativas. Conocer las potencialidades y las fronteras de cada una constituye una condición necesaria para el diseño de mejores entornos de aprendizaje. 


\section{Referencias}

Alcaraz, F. G., Espín, A. A., Martínez, A. H., \& Alarcón, M. M. (2006). Diseño de cuestionarios para la recogida de información: metodología y limitaciones. Revista clinica de medicina de familia, 1(5), 232-236.

Bergmann, J., \& Sams, A. (2012). Flip your classroom: Reach every student in every class every day. International Society for Technology in Education.

Failache, E., Katzkowicz, N., \& Machado, A. (2020). La Educación en Tiempos de Pandemia y el Día Después: El Caso de Uruguay. Revista Internacional De Educación Para La Justicia Social, 9(3). https:// revistas.uam.es/riejs/article/view/12185

García Aretio, L. (1999). Fundamento y componentes de la educación a distancia. Revistalberoamericana de Educación a Distancia, 2(2), 43-61.

Graham, C. R. (2006). Blended learning systems. The handbook of blended learning, 3-21.

INEEd (2017). Informe sobre el estado de la educación en Uruguay 2015-2016. http://www.ineed.edu. uy/images/pdf/Informe-sobre-el-estado-de-laeducacion-en-Uruguay-2015-2016.pdf

Mills, G. E. (2003). Action Research. A Guide for the Teacher Researcher. Soutern Oregon University.

Sagarra, S. (2018). Contando visitas web: ¿es posible aplicar modelos de Aula Invertida en Educación Media Superior? Revista Educación en Ciencias Biológicas, 3(1), 49-56.
Salinas, I. (2011). Entornos virtuales de aprendizaje en la escuela: tipos, modelo didáctico y rol del docente. Pontificia Universidad Católica Argentina. https://tecnologiaaplicadaeducacionurn. files.wordpress.com/2012/09/salinas_2011_ entornosvirtualesaprendizaje.pdf

Salomon, G. (2002). La educación superior frente a los desafíos de la era de la información. Boletín de la Red Estatal de Docencia Universitaria, 2(2), 5-11.

Santos, G., Ferran, N., \& Abadal, E. (2012). Recursos educativos abiertos: repositorios y uso. $E I$ Profesional de La Información, 21(2), 136-145.

Ferro Soto, C., Martínez Senra, A. I., \& Otero Neira, M. C. (2009). Ventajas del uso de las TICs en el proceso de enseñanza-aprendizaje desde la óptica de los docentes universitarios españoles. Edutec. Revista Electrónica De Tecnología Educativa, (29), a119. https://doi.org/10.21556/edutec.2009.29.451

Viner, R. M., Russell, S. J., Croker, H., Packer, J., Ward, J., Stansfield, C., Mytton, O., Bonell, C., \& Booy, R. (2020). School closure and management practices during coronavirus outbreaks including COVID-19: a rapid systematic review. The Lancet. Child \& adolescent health, 4(5), 397-404. https://doi. org/10.1016/S2352-4642(20)30095-X 


\section{Anexos}
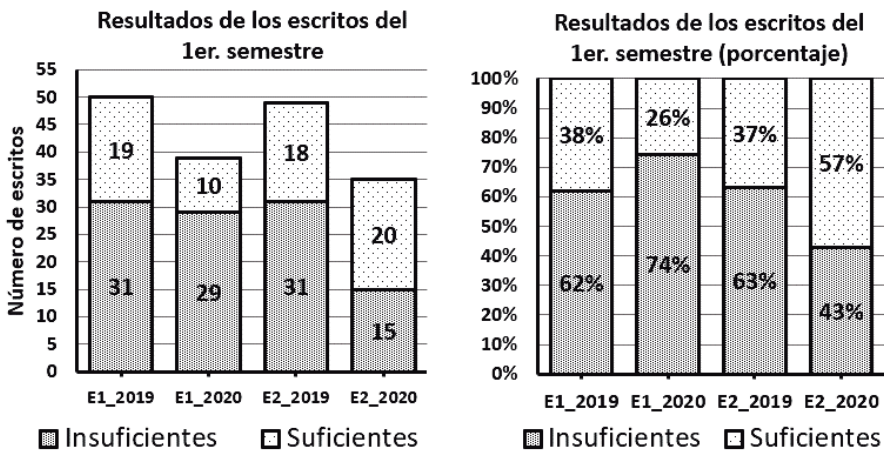

Figura 1.Resultado de la primera y segunda prueba escrita (E1 y E2) de los años 2019 y 2020. A la izquierda se muestran los números absolutos de escritos realizados, discriminados por suficientes (punteado laxo) e insuficientes (punteado denso). A la derecha se describen las mismas entregas, pero expresadas en porcentaje de suficientes e insuficientes.

Tabla 1. Pruebas de $\chi^{2}$ de $2 \times 2$ comparando la realización (y suficiencia) de las pruebas escritas del primer semestre 2019 y 2020. El test responde a la cuestión de si las diferencias en cantidad de escritos realizados (o de suficiencias alcanzadas) entre los dos años (figura 1) se atribuye a deriva aleatoria o a diferencias entre los grupos considerados. Un resultado significativo se interpreta como improbablemente sustentado en fenómenos estocásticos.

\begin{tabular}{|l|c|c|c|c|}
\hline & \multicolumn{3}{|c|}{ Resultados de los test $\chi^{2}$} \\
\hline Comparación & $\chi^{2}$ & N & Grados de libertad & Significación (dos colas) \\
\hline Realización del 1er. Escrito 2019 vs. 2020 & 7.58 & 129 & $\mathbf{1}$ & $\mathbf{0 , 0 0 5 9 *}$ \\
\hline Realización del 2do. Escrito 2019 vs. 2020 & 10.18 & 129 & $\mathbf{1}$ & $\mathbf{0 , 0 0 1 4}$ \\
\hline Suficiencia del 1er. Escrito 2019 vs. 2020 & 1,52 & 89 & $\mathbf{1}$ & 0,2171 \\
\hline Suficiencia del 2do. Escrito 2019 vs. 2020 & 3,43 & 84 & $\mathbf{1}$ & $\mathbf{0 , 0 6 3 9}$ \\
\hline
\end{tabular}

* Valores estadísticamente significativos $(p<0,05)$.

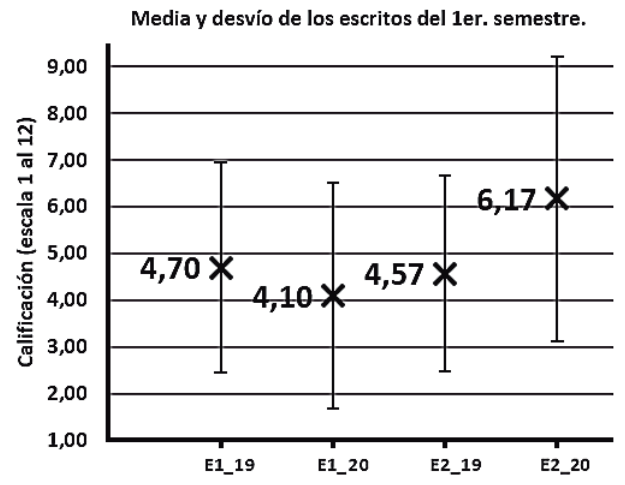

Figura 2: Comparación de la calificación promedio de las pruebas escritas del primer semestre. Se muestran las primeras pruebas 2019 y 2020 (fin de abril) y luego las segundas pruebas 2019 y 2020 (fin de junio). Las barras indican (+/-) una desviación estándar. En una distribución normal dicho rango comprende el $68 \%$ del total de casos. 
Tabla 2. Pruebas $t$ de student. Se comparan las medias de las pruebas escritas 2019 y 2020 (muestras independientes) y del primero y segundo escrito de cada año (muestras relacionadas). En el último caso solo se consideran los estudiantes que realizaron ambas evaluaciones. Un resultado significativo indica que la probabilidad que la diferencia entre las medias se deba al azar es muy baja.

\begin{tabular}{|c|c|c|c|c|c|c|}
\hline \multirow{2}{*}{\multicolumn{2}{|c|}{$\begin{array}{c}\text { Comparación } \\
\text { N }\end{array}$}} & \multicolumn{5}{|c|}{ Resultados de los test_t } \\
\hline & & \multirow{2}{*}{$\frac{M(S D)}{50}$} & \multirow{2}{*}{$\begin{array}{c}\text { Grados de libertad } \\
4,70(2,26)\end{array}$} & \multirow{3}{*}{87} & \multirow{3}{*}{$\begin{array}{c}\text { Significación (dos colas) } \\
1,2787\end{array}$} & \multirow{3}{*}{0,2044} \\
\hline \multirow{2}{*}{ E1 } & 2019 & & & & & \\
\hline & 2020 & 39 & $4,10(2,09)$ & & & \\
\hline \multirow{2}{*}{ E2 } & 2019 & 49 & $4,57(2,42)$ & \multirow{2}{*}{82} & \multirow{2}{*}{$-2,6794$} & \multirow{2}{*}{$0,0089 *$} \\
\hline & 2020 & 35 & $6,17(3,04)$ & & & \\
\hline \multirow{2}{*}{2019} & E1 & 46 & $4,87(2,24)$ & \multirow{2}{*}{45} & \multirow{2}{*}{$-0,4121$} & \multirow{2}{*}{0,6822} \\
\hline & E2 & 46 & $4,70(2,45)$ & & & \\
\hline \multirow{2}{*}{2020} & E1 & 29 & $4,38(2,21)$ & \multirow{2}{*}{28} & \multirow{2}{*}{4,1366} & \multirow{2}{*}{$0,0003^{*}$} \\
\hline & E2 & 29 & $6,28(2,95)$ & & & \\
\hline
\end{tabular}

* Valores estadísticamente significativos $(p<0,05)$.

Tabla 3. Respuestas del cuestionario. Se apuntó a la exhaustividad y la sistematización de las opiniones recogidas. Entre paréntesis se indica las veces que se reiteró el concepto (si fue más de una).

\begin{tabular}{l} 
Pregunta \\
\hline - ¿Cuántos cuestionarios semanales realizó? \\
- Registro tardío en EVA (2) \\
- Problemas técnicos al realizarlo (2) \\
- Problemas temporales con Internet (4) \\
- Dificultades de comprensión (2) \\
- Olvido, falta de dedicación (2) \\
- Falta de tiempo. \\
- ¿'Tuvo inconvenientes para seguir el curso virtual? \\
- Problemas transitorios con Internet (4) \\
- Problemas de acceso al EVA (4) \\
- Carencia de dispositivo fijo de acceso \\
- Dificultades de comprensión de textos \\
- Ausencia de hábitos de trabajo autónomo \\
- Falta de constancia para seguir el curso \\
- Interferencia con otras actividades (2)
\end{tabular}




\begin{tabular}{|c|c|}
\hline Pregunta & Proporcio \\
\hline 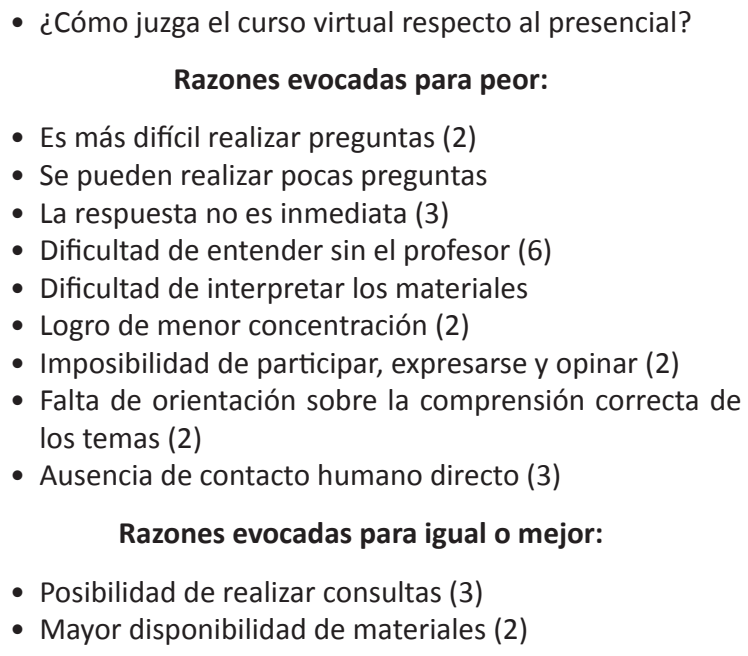 & $\begin{array}{l}\text { - Materiales bien explicados } \\
\text { - Los videos ayudan a aprender } \\
\text { - Mayor tiempo para estudiar, repasar, tomar apuntes (4) } \\
\text { - Permite estudiar c/más tranquilidad (4) } \\
\text { - Permite que la familia participe } \\
\text { - Facilita a estudiantes introvertidos } \\
\text { - Experiencia previa en cursos virtuales }\end{array}$ \\
\hline $\begin{array}{l}\text { - ¿Cuál alternativa prefiere a la presencialidad? } \\
\text { (VC: videoconferencia, WA: WhatsApp) } \\
\text { Razones evocadas para preferir un EVA: } \\
\text { - Permite mejor autoorganización (3) } \\
\text { - El curso es más organizado (4) } \\
\text { - No se pierden temas al inasistir } \\
\text { - Promueve los hábitos de estudio } \\
\text { - No interfiere con otras labores } \\
\text { - Las consultas quedan disponibles para todos } \\
\text { - Materiales bien explicados } \\
\text { - Funcionamiento sencillo } \\
\text { - Escasa asistencia a las VC } \\
\text { Razones evocadas para WA o mail: } \\
\text { - Mejor comunicación } \\
\text { - Mayor rapidez } \\
\text { - Mayor organización }\end{array}$ & 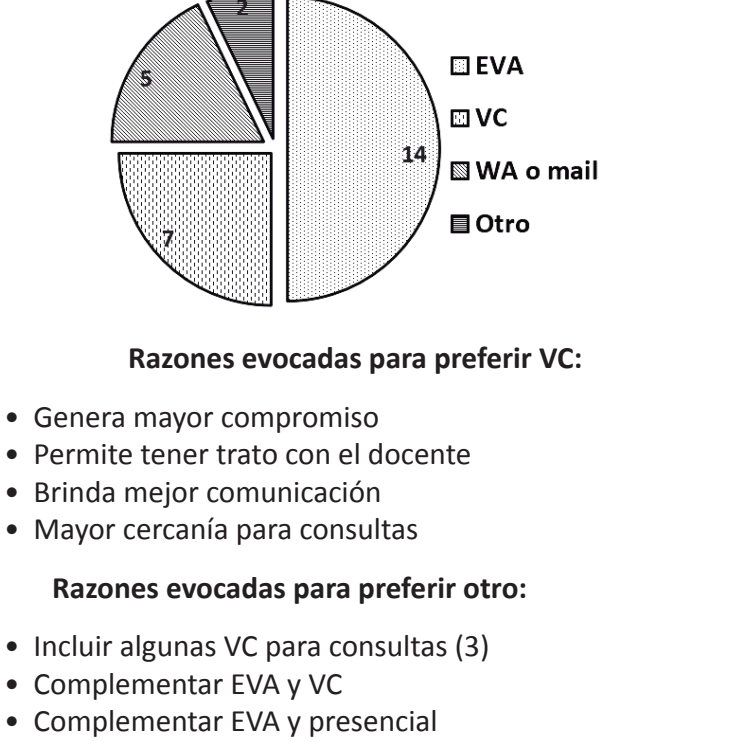 \\
\hline
\end{tabular}

